Utah State University

DigitalCommons@USU

$1-1-2006$

\title{
Modeled vs. Actual Performance of the Geosynchronous Imaging Fourier Transform Spectrometer (GIFTS)
}

Gregory W. Cantwell

John D. Elwell

Roy W. Esplin

Mark P. Esplin

Deron K. Scott

Lorin J. Zollinger

See next page for additional authors

Follow this and additional works at: https://digitalcommons.usu.edu/sdl_pubs

\section{Recommended Citation}

Cantwell, Gregory W.; Elwell, John D.; Esplin, Roy W.; Esplin, Mark P.; Scott, Deron K.; Zollinger, Lorin J.; Bingham, Gail E.; Revercomb, Henry E.; Smith, William L.; and Reisse, Robert A., "Modeled vs. Actual Performance of the Geosynchronous Imaging Fourier Transform Spectrometer (GIFTS)" (2006). Space Dynamics Lab Publications. Paper 27.

https://digitalcommons.usu.edu/sdl_pubs/27

This Article is brought to you for free and open access by the Space Dynamics Lab at DigitalCommons@USU. It has been accepted for inclusion in Space Dynamics Lab Publications by an authorized administrator of DigitalCommons@USU. For more information, please contact digitalcommons@usu.edu.

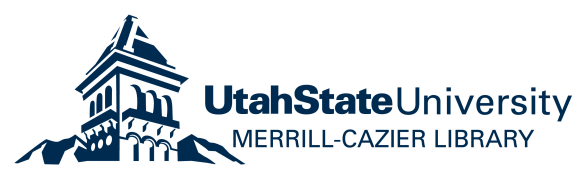




\section{Authors}

Gregory W. Cantwell, John D. Elwell, Roy W. Esplin, Mark P. Esplin, Deron K. Scott, Lorin J. Zollinger, Gail E. Bingham, Henry E. Revercomb, William L. Smith, and Robert A. Reisse 
Cantwell, Gregory W., John D. Elwell, Roy W. Esplin, Mark P. Esplin, Deron K. Scott, Lorin J. Zollinger, Gail E. Bingham, Henry E. Revercomb, William L. Smith, and Robert A. Reisse. 2006. "Modeled vs. Actual Performance of the Geosynchronous Imaging Fourier Transform Spectrometer (GIFTS)." Proceedings of SPIE 6297, 62970T. doi:10.1117/12.684136.

\title{
Modeled vs. actual performance of the geosynchronous imaging Fourier transform spectrometer (GIFTS)
}

\author{
Gregory W. Cantwell ${ }^{*}$, John D. Elwell ${ }^{\mathrm{a}}$, Roy W. Esplin ${ }^{\mathrm{a}}$, Mark P. Esplin ${ }^{\mathrm{a}}$, Deron K. Scott ${ }^{\mathrm{a}}$, Lorin J. \\ Zollinger ${ }^{\mathrm{a}}$, Gail E. Bingham ${ }^{\mathrm{a}}$, Henry E. Revercomb ${ }^{\mathrm{b}}$, William L. Smith ${ }^{\mathrm{c}, \mathrm{b}}$, Robert A. Reisse ${ }^{\mathrm{d}}$ \\ ${ }^{a}$ Space Dynamics Laboratory, Utah State University, Logan, UT \\ ${ }^{\mathrm{b}}$ Space Science and Engineering Center, University of Wisconsin-Madison, Madison, WI \\ ${ }^{\mathrm{c}}$ Center for Atmospheric Sciences, Hampton University, Hampton, VA \\ ${ }^{\mathrm{d}}$ NASA Langley Research Center, Hampton, VA
}

\begin{abstract}
The NASA Geosynchronous Imaging Fourier Transform Spectrometer (GIFTS) has been completed as an Engineering Demonstration Unit (EDU) and has recently finished thermal vacuum testing and calibration. The GIFTS EDU was designed to demonstrate new and emerging sensor and data processing technologies with the goal of making revolutionary improvements in meteorological observational capability and forecasting accuracy. The GIFTS EDU includes a cooled $(150 \mathrm{~K})$, imaging FTS designed to provide the radiometric accuracy and atmospheric sounding precision required to meet the next generation GOES sounder requirements. This paper discusses a GIFTS sensor response model and its validation during thermal vacuum testing and calibration.

The GIFTS sensor response model presented here is a component-based simulation written in IDL with the model component characteristics updated as actual hardware has become available. We discuss our calibration approach, calibration hardware used, and preliminary system performance, including NESR, spectral radiance responsivity, and instrument line shape. A comparison of the model predictions and hardware performance provides useful insight into the fidelity of the design approach.
\end{abstract}

Keywords: GIFTS, system performance, radiometric modeling, Fourier transform spectrometer (FTS), remote sensing

\section{INTRODUCTION}

The NASA Geosynchronous Imaging Fourier Transform Spectrometer (GIFTS) Sensor Module (SM) has been completed as an Engineering Demonstration Unit (EDU) and has recently finished thermal vacuum testing and calibration. The GIFTS EDU was designed and fabricated by the Space Dynamics Laboratory at Utah State University (SDL/USU) to demonstrate new and emerging sensor and data processing technologies with the goal of making revolutionary improvements in meteorological observational capability and forecasting accuracy ${ }^{1,2}$. The GIFTS EDU includes a cryogenically cooled imaging Fourier transform spectrometer (FTS) with programmable spectral resolution designed to provide the radiometric accuracy and atmospheric sounding precision required to meet the next generation GOES sounder requirements. In this paper a GIFTS sensor response model is described, and both modeled and preliminary actual performance of the GIFTS EDU are presented.

Figure 1 shows a layout of the GIFTS optical system. Radiation entering the system is directed by the pointing mirror (PM) into the telescope (fore optics). An IR/visible dichroic separates the radiation into visible and infrared (IR) bands. The visible radiation is focused on the active pixel sensor (APS). The IR radiation is reflected into the FTS, which is a plane-mirror Michelson interferometer. After passing through the FTS the IR radiation is directed into the aft optics where an IR dichroic beamsplitter separates the IR radiation into the long-wave IR (LWIR) band $\left(685-1130 \mathrm{~cm}^{-1}\right)$ and the short/mid-wave IR (SMWIR) band $\left(1650-2250 \mathrm{~cm}^{-1}\right)$. The IR radiation in these two bands is then focused on the LWIR and SMWIR focal plane arrays (FPAs). A flip-in mirror can be inserted into the optical path between M2 and M3

* greg.cantwell@sdl.usu.edu; phone: 435 797-4668

Infrared Spaceborne Remote Sensing XIV, edited by Marija Strojnik, Proc. of SPIE Vol. 6297, 62970T, (2006) · 0277-786X/06/\$15 - doi: 10.1117/12.684136 
to allow the FPAs to view one of two internal blackbodies designed by the University of Wisconsin-Madison Space Science and Engineering Center ${ }^{3,4}$. A cold shutter at $60 \mathrm{~K}$ can be moved into the optical path in front of the Lyot stop to block all incoming radiation to make measurements of the detector dark offset levels. An on-board miniature cryocooler cools the FTS to $150 \mathrm{~K}$ and the FPAs to $60 \mathrm{~K}$. A stabilized $1064.57 \mathrm{~nm}$ diode pumped laser provides the optical frequency reference for the FTS. The GIFTS spectral resolution is programmable with a maximum spectral resolution of $0.57 \mathrm{~cm}^{-1}$. Each IR FPA contains $128 \times 128$ individual detector elements that have a single-pixel spatial geometric footprint of $4 \times 4 \mathrm{~km}$. The total FPA footprint is $512 \times 512 \mathrm{~km}$.

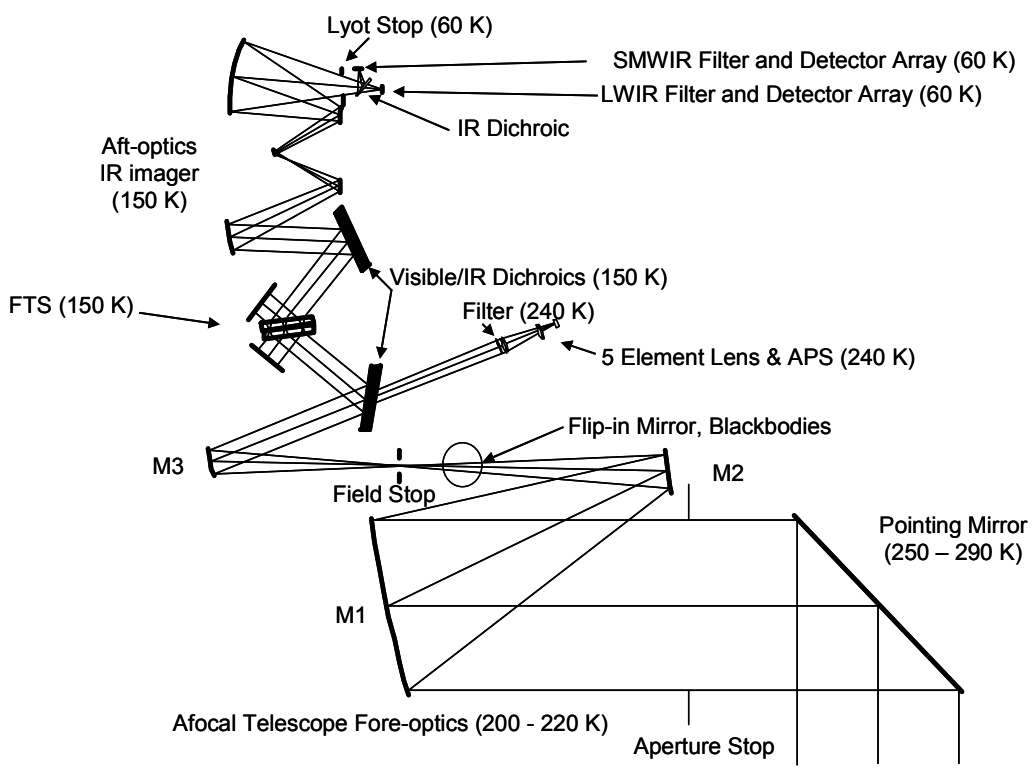

Fig. 1. GIFTS optical diagram.

\section{GIFTS SENSOR RESPONSE MODEL}

The GIFTS sensor response model is written in the IDL programming language and is intended to give a complete and accurate first-order simulation of the GIFTS response to a given input spectral radiance. A block diagram of the model is shown in Figure 2. The units at each stage of the simulation are given below the respective quantities. The model is constructed to simulate the physical hardware as closely as possible, and has been refined and updated as actual hardware characteristics have become available.

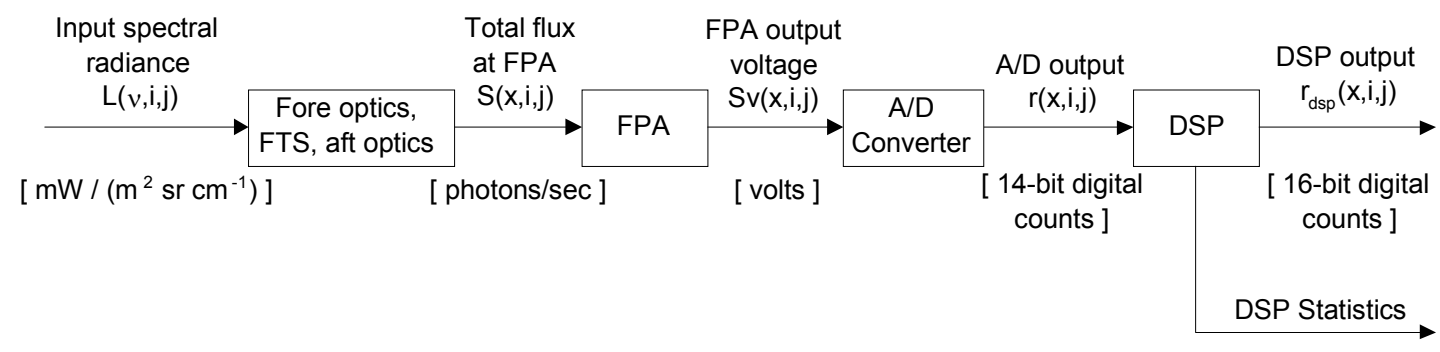

Fig. 2. GIFTS sensor response model block diagram. 
The input to the model is the spectral radiance for a particular pixel $L(v, i, j)$, where $v$ is the wavenumber vector and $i, j$ are the pixel coordinates. The first block models the GIFTS fore optics, FTS, and aft optics. The output of the first block, $S(x, i, j)$, is the total flux incident on the FPA as a function of optical path difference (OPD), $x$, and the pixel coordinates. $S(x, i, j)$ accounts for the instrument throughput and includes photon contributions from the scene and GIFTS optics. Spectral optical efficiencies are used for the pointing mirror, M1, M2, M3, the FTS beamsplitter/compensator, and the optical bandpass filter. Lumped-element, band-averaged optical efficiencies are used for all other optical elements. Band-averaged single-element emittances are used for the pointing mirror, the IR dichroic beamsplitter and the optical bandpass filter. Lumped-element, band-averaged effective emittances are used for all other optics. The interferogram $S(x, i, j)$ is a non-ideal interference term accounting for non-unity modulation efficiency and non-zero phase due to instrument effects such as beamsplitter-compensator mismatch. The interferogram includes OPD sampling interval differences for off-axis pixels, the proper number of samples, and the proper spectral resolution. Interferograms with modulation efficiencies that vary as a function of both wavenumber and OPD due to tilt of the interferometer moving mirror can be modeled using a mirror tilt term that is a function of OPD.

The FPA block in Figure 2 models FPA pixel element $(i, j)$ and the FPA read-out integrated circuit (ROIC). Pixel-topixel non-uniformities in detector resistance-area product $\left(R_{0} A\right)$ and peak quantum efficiency are modeled, as are the resulting pixel-to-pixel non-uniformities in dark current, detector noise, and responsivity. Nominal $R_{0} A$ and quantum efficiency values, detector relative spectral responses, and detector nonlinearity are based on preliminary estimates or measurements by the FPA manufacturer. Finite FPA electron-well depths are modeled. Nominal FPA fixed-integration times are used. Photon noise, FPA thermal noise, and ROIC noise are all modeled.

The A/D converter block in Figure 2 models the analog-to-digital converter (ADC). In the actual GIFTS EDU the LWIR FPA has 16 output taps and the SMWIR FPA has 4 output taps, with one ADC per tap. The model simulates a single 14-bit ADC per FPA and includes ADC electronics noise.

The DSP block in Figure 2 simulates the digital signal processor (DSP), which for the GIFTS EDU was implemented in software. The DSP functions include co-adding of samples in the LWIR channel, computation of statistics prior to digital filtering, digital filtering, and decimation. The digital filter coefficients are complex and the output of the DSP, $r_{d s p}(x, i, j)$, is a complex interferogram as a function of OPD and the pixel coordinates.

The model can output the response at one of three possible points in the signal chain in Figure 2: the output of the FPA, the output of the A/D, or the output of the DSP. The model is capable of simulating the GIFTS response as full 128 $\times 128$ interferogram data cubes for both IR channels, or single interferograms for a given pixel from either FPA. The non-ideal instrument effects such as noise, nonlinearity, FPA non-uniformities, and interferometer mirror tilt can be switched on or off when the model is run, as desired by the user. Table 1 lists the model parameters and their default values.

The model has been a powerful tool during instrument design, calibration planning, thermal vacuum testing, and calibration. During instrument design the model was used to assess the impact of expected tilt of the interferometer moving mirror as a function of OPD on noise equivalent spectral radiance (NESR), with the conclusion that expected tilts would have negligible impact on NESR. It was used to simulate the effect of a FPA read-out anomaly as interferograms were propagated through the filtering and decimation stages of the digital signal processor (DSP), to assess the impact of the anomaly on NESR, and the effectiveness of a possible correction for the anomaly. The model was also used in the development and testing of a proposed linearity correction algorithm for the filtered/decimated interferograms. During calibration planning the model was used to generate full GIFTS synthetic data cubes that were used in development and testing of calibration software as well as to simulate GIFTS responses to various calibration configurations. The model was used during thermal vacuum testing and calibration to assist in troubleshooting and confirmation of expected responses. 
Table 1. GIFTS sensor response model parameters.

\begin{tabular}{|c|c|c|c|c|}
\hline Parameter & LWIR Value & SMWIR Value & Units & Description/Notes \\
\hline $\mathrm{A} \Omega$ & $5.652 \mathrm{E}-10$ & $5.652 \mathrm{E}-10$ & $\mathrm{~m}^{2} \mathrm{sr}$ & throughput (per-pixel) \\
\hline $\mathrm{T}_{\mathrm{pm}}$ & 250 & 250 & $\mathrm{~K}$ & temperature of the pointing mirror $(\mathrm{PM})$ \\
\hline$\varepsilon_{\mathrm{pm}}$ & 0.037 & 0.012 & unitless & emittance of pointing mirror \\
\hline $\max \left[\tau_{\mathrm{pm}}(v)\right]$ & 0.985 & 0.990 & unitless & peak optical efficiency of pointing mirror \\
\hline $\mathrm{T}_{1}$ & 220 & 220 & $\mathrm{~K}$ & temperature of telescope fore-optics region (M1, M2, M3) \\
\hline$\varepsilon_{1}$ & 0.027 & 0.027 & unitless & effective emittance of telescope fore-optics \\
\hline $\max \left[\tau_{1}(v)\right]$ & 0.963 & 0.966 & unitless & peak optical efficiency of fore-optics \\
\hline $\mathrm{T}_{2}$ & 150 & 150 & $\mathrm{~K}$ & $\begin{array}{l}\text { temperature of aft-optics region (D1, FTS, D2, M4, M5, } \\
\text { M6, M7, M8) }\end{array}$ \\
\hline$\varepsilon_{2}$ & 0.086 & 0.087 & unitless & effective emittance of aft-optics \\
\hline $\max \left[\tau_{2}(v)\right]$ & 0.321 & 0.318 & unitless & peak optical efficiency of aft-optics \\
\hline $\mathrm{T} 3$ & 60 & 60 & $\mathrm{~K}$ & temperature of IR FPA region (D3, optical filter, FPA) \\
\hline$\varepsilon_{\mathrm{D} 3}$ & 0 & 0 & unitless & emittance of D3 \\
\hline$\tau_{\mathrm{D} 3}$ & 0.83 & 0.999 & unitless & optical efficiency of D3 \\
\hline$\varepsilon_{\mathrm{opf}}$ & 0.222 & 0.111 & unitless & emittance of bandpass optical filter \\
\hline $\max \left[\tau_{\text {opf }}(v)\right]$ & 0.890 & 0.954 & unitless & peak optical efficiency of bandpass optical filter \\
\hline$\tau_{\text {det }}$ & 0.97 & 0.97 & unitless & optical efficiency of detector AR coating \\
\hline $\mathrm{N}_{\mathrm{ifg}}$ & 66276 & 16520 & unitless & number of raw interferogram samples \\
\hline$\lambda_{\text {laser }}$ & 1064.57 & 1064.57 & $\mathrm{~nm}$ & metrology laser wavelength \\
\hline $\mathrm{d} v$ & 0.57 & 0.57 & $\mathrm{~cm}^{-1}$ & spectral resolution (on-axis) \\
\hline$\zeta$ & 0.73 & 0.79 & unitless & modulation efficiency \\
\hline$\theta_{\text {pix }}$ & 766.5 & 766.5 & $\mu \mathrm{rad}$ & $\begin{array}{l}\text { angle subtended by the width of one pixel at interferometer } \\
\text { mirrors }\end{array}$ \\
\hline$v_{\text {low }}$ & 685 & 1650 & $\mathrm{~cm}^{-1}$ & low wavenumber cutoff for measurement band \\
\hline$v_{\text {high }}$ & 1130 & 2250 & $\mathrm{~cm}^{-1}$ & high wavenumber cutoff for measurement band \\
\hline $\mathrm{R}_{\mathrm{m}}$ & 1.75 & 1.75 & $\mathrm{~cm}$ & beam radius at interferometer mirrors \\
\hline$\phi_{\text {tilt }}$ & 3.3 & 3.3 & $\operatorname{arcsec}$ & maximum mirror tilt (at ends of high resolution scan) \\
\hline $\mathrm{F}_{\mathrm{h}}$ & 128 & 128 & unitless & FPA height in pixels \\
\hline $\mathrm{F}_{\mathrm{w}}$ & 128 & 128 & unitless & FPA width in pixels \\
\hline$A_{d}$ & $3.60 \mathrm{E}-09$ & $3.60 \mathrm{E}-09$ & $\mathrm{~m}^{2}$ & detector pixel area \\
\hline wd & $100 \mathrm{E}+06$ & $20 \mathrm{E}+06$ & unitless & FPA detector well depth in electrons \\
\hline $\mathrm{qe}_{\text {nom }}$ & 0.9 & 0.9 & unitless & nominal peak detector quantum efficiency \\
\hline $\mathrm{qe}_{\text {std }}$ & 0.05 & 0.05 & unitless & pixel-to-pixel std. dev. of peak quantum efficiency \\
\hline $\mathrm{R}_{0} \mathrm{~A}_{\text {nom }}$ & 41 & $5.0 \mathrm{E}+05$ & $\mathrm{ohm} \mathrm{cm}^{2}$ & nominal detector $\mathrm{R}_{0} \mathrm{~A}$ product \\
\hline $\mathrm{R}_{0} \mathrm{~A}_{\text {std }}$ & 5 & $1.0 \mathrm{E}+05$ & ohm cm ${ }^{2}$ & pixel-to-pixel std. dev. of $\mathrm{R}_{0} \mathrm{~A}$ product \\
\hline $\mathrm{v}_{\mathrm{b}}$ & -20.0 & -20.0 & mvolts & detector bias voltage \\
\hline $\mathrm{t}_{\text {int }}$ & 134 & 545 & $\mu \mathrm{sec}$ & nominal detector fixed-integration time \\
\hline$t_{\text {samp }}$ & 166.5 & 666.5 & $\mu \mathrm{sec}$ & nominal FPA sample time \\
\hline$\sigma_{\text {ROIC }}$ & 14564 & 1781 & unitless & ROIC noise electrons \\
\hline$v_{\max }$ & 2.7 & 2.7 & volts & FPA output voltage for a full well \\
\hline $\mathrm{AD}_{\text {bits }}$ & 14 & 14 & unitless & number of A/D bits \\
\hline $\mathrm{AD}_{\min }$ & 0 & 0 & volts & the minimum value defining the $\mathrm{A} / \mathrm{D}$ dynamic range \\
\hline $\mathrm{AD}_{\max }$ & 2.7 & 2.7 & volts & the maximum value defining the $\mathrm{A} / \mathrm{D}$ dynamic range \\
\hline$\sigma_{\mathrm{AD}}$ & 165 & 165 & $\mu$ volts & $\mathrm{A} / \mathrm{D}$ electronics noise \\
\hline
\end{tabular}




\section{GIFTS EDU CALIBRATION}

The approach used by SDL to calibrate a radiometric sensor involves characterizing the sensor in several domains, including the sensor's radiometric responsivity, spectral responsivity, spatial responsivity, temporal responsivity, and polarization responsivity ${ }^{5}$. Ideally the sensor response in each of these domains is characterized independently of the others; however, compete independence is not always possible. A complete calibration characterizes the sensor in each of these domains and includes estimates of measurement uncertainties. SDL has traditionally grouped calibration parameters into two groups. In the first group are those parameters used in the calibration equation to convert sensor output to engineering units. The second group includes all other parameters not included in the calibration equation but still needed for a complete characterization of the instrument, such as NESR. During the calibration of the GIFTS EDU the instrument was characterized in all of the domains with the exception of polarization responsivity ${ }^{6}$, which was eliminated due to project funding limitations.

Two primary calibration sources were used during calibration of the GIFTS EDU: the $2^{\text {nd }}$ multifunction infrared calibrator (MIC2) ${ }^{7}$ and the 15 -inch high accuracy extended source (HAES-15) ${ }^{8}$. These sources, pictured in Figure 3, can be attached to the GIFTS thermal vacuum test chamber and, when attached, share a common vacuum with it. The MIC2 includes an extended area blackbody source, and can be used in collimator mode, Jones source mode, or scatter source mode with a variety of external laboratory sources including external blackbodies, gas cells, and lasers. The MIC 2 also includes several optical filters and various sized apertures that may be moved in or out of the optical path at the entrance aperture. The HAES-15 is a high emissivity extended area blackbody source with traceability to NIST.

During GIFTS EDU calibration the MIC2 was used in collimator mode with an external blackbody and various aperture sizes to perform measurements for the spatial calibration, including point source responses and optical distortion. The MIC2 was used in collimator mode with an IR laser and gas cells to perform measurements for the spectral calibration, including line position and instrument line shape. The MIC2 was also used to perform system level measurements of the GIFTS EDU nonlinearity using the small signal linearity technique. The HAES-15 was used to perform radiance responsivity measurements throughout the dynamic range. The HAES-15 was also used as a cold $(\sim 80 \mathrm{~K})$ external source to make measurements of the emissions from the telescope optics.

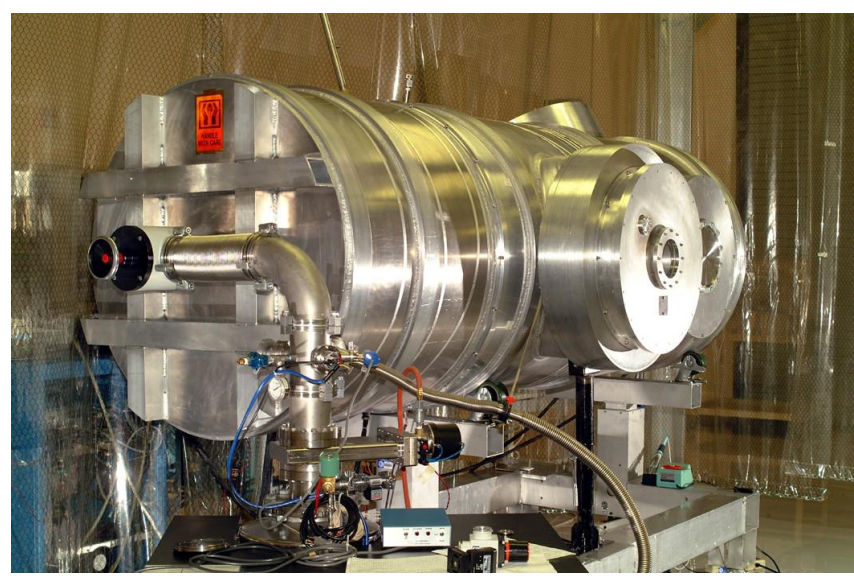

MIC2

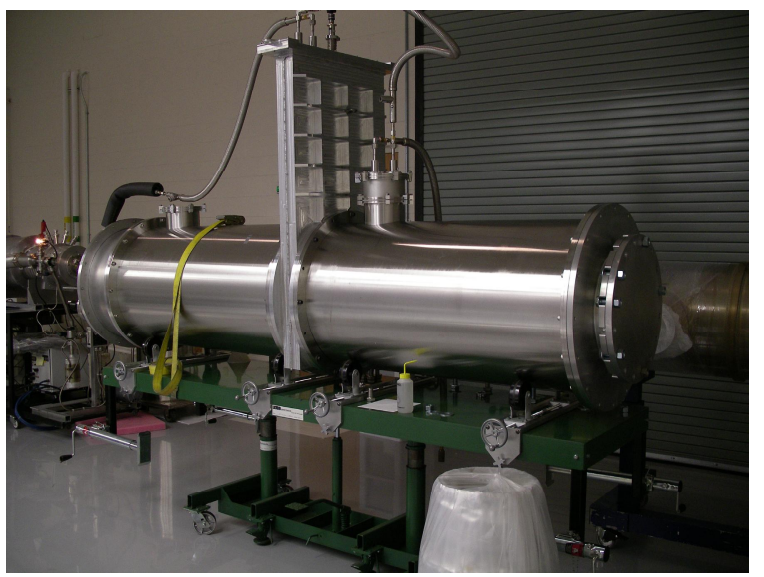

HAES-15

Fig. 3. The MIC2 and HAES-15 calibrators were used in the GIFTS EDU calibration.

\section{COMPARISON OF MODELED AND ACTUAL PERFORMANCE}

Preliminary GIFTS EDU performance results were compared with results predicted by the GIFTS sensor response model for the following performance measures and instrument characteristics: spectral radiance responsivity (SRR), 
noise equivalent spectral radiance (NESR), instrument line shape, out-of-band nonlinear harmonics, and apparent line shifts for off-axis pixels.

Pixels with nominal responsivities and noise from each FPA were selected for the SRR and NESR comparisons. Fig. 4 shows histograms of the integrated responsivity and noise for each FPA and the pixels selected for the comparisons. Pixels are indexed using the convention (column, row) where pixel $(0,0)$ is in the upper left corner of the FPA.
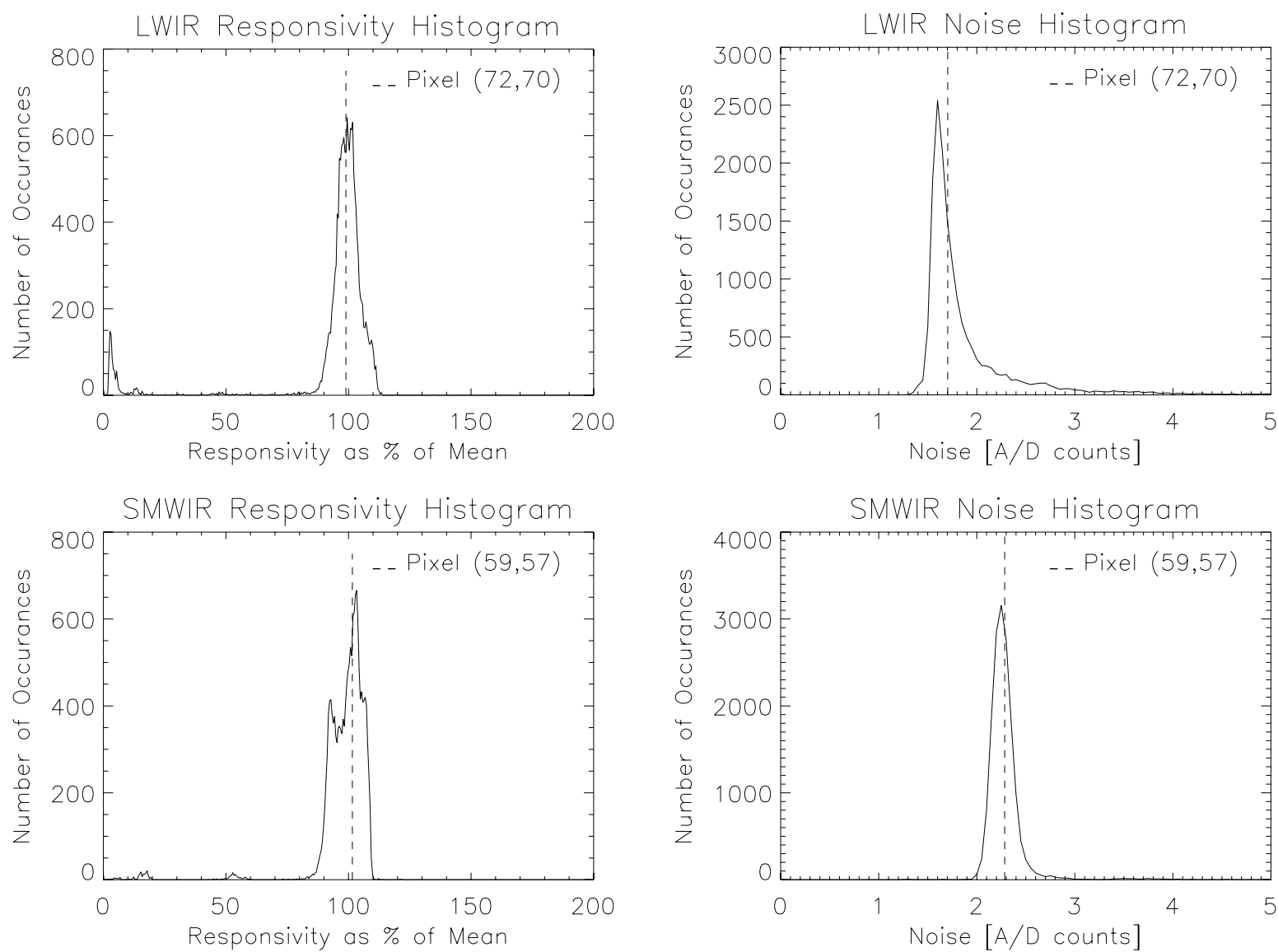

Fig. 4. Histograms of the GIFTS EDU integrated responsivity and noise from the LWIR and SMWIR FPAs. Pixel (72,70) from the LWIR FPA and pixel $(59,57)$ from the SMWIR FPA, which were used for SRR and NESR comparisons, are shown to be nominal pixels in both responsivity and noise.

Fig. 5 shows the modeled and preliminary measured SRR. The measured SRR was computed as follows: individual spectra with the HAES-15 extended area blackbody source at two different source temperatures were computed and phase corrected, and the real part of the phase corrected spectra were averaged for each temperature. The SRR was computed as the ratio of the difference of the average spectra to the difference in the Planck functions at the two source temperatures. No linearity correction was applied when computing the preliminary SRR. The modeled SRR was computed by a complete end-to-end simulation. Modeled linear, noise-free GIFTS interferograms were computed using Planck functions at the two HAES-15 source temperatures as model inputs. The simulated interferograms were then processed with identical processing steps used to compute the measured SRR. 
There is good general agreement between the modeled and actual SRR. The discrepancy between modeled and actual results in the LWIR band near $800 \mathrm{~cm}^{-1}$ may be due to the fact that the model does not have spectral transmission values for many of the optics, polarization effects have not been included in the model, and/or linearity correction has not been performed on the measured results.
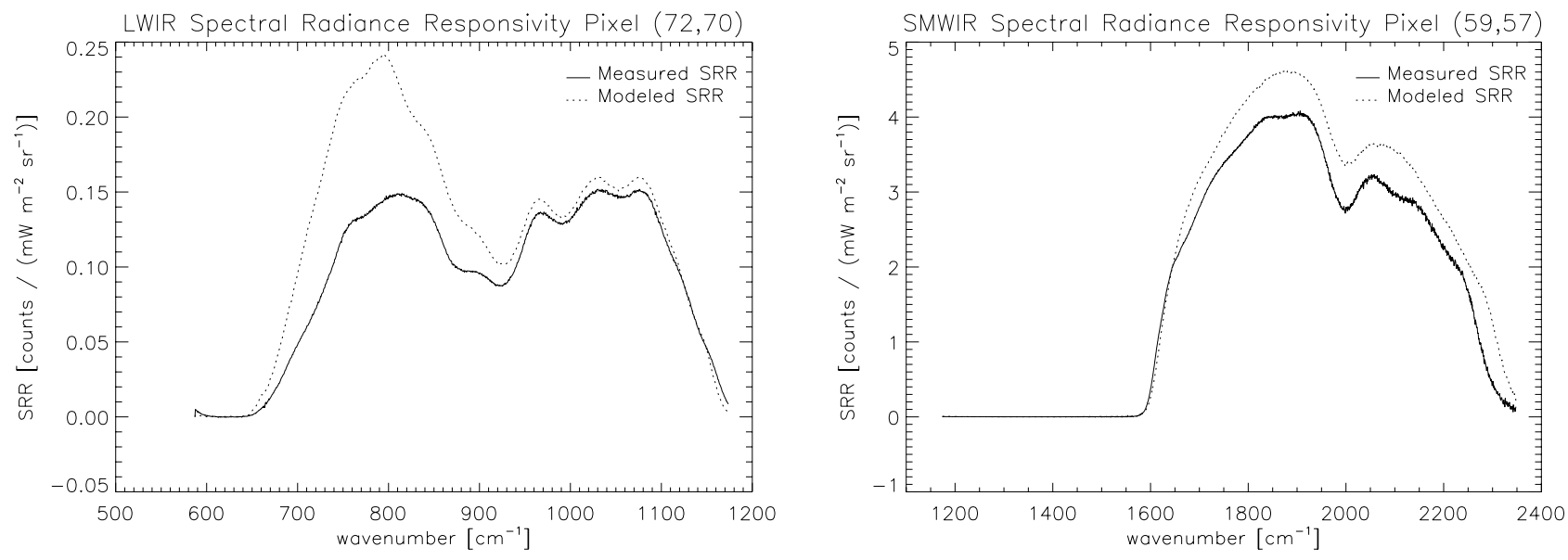

Fig. 5. Comparison of GIFTS EDU measured and modeled spectral radiance responsivity (SRR) for nominal pixels from the LWIR and SMWIR FPAs.

Fig. 6 compares the modeled and measured NESR, which is a measure of the spectral radiance uncertainty associated with a single GIFTS measurement. The measured NESR estimates were calculated as follows. The spectral standard deviation of several individual phase corrected spectra was computed as an estimate of the spectral noise associated with a single measurement. The spectral noise estimates for the LWIR and SMWIR bands were computed from spectra taken while viewing the HAES-15 at source temperatures of $276 \mathrm{~K}$ and $260 \mathrm{~K}$, respectively, because these are the temperatures at which the GIFTS EDU NESR requirements are specified. The spectral noise estimate was then converted to radiance units by dividing it by the SRR. The modeled NESR was generated by computing modeled interferograms, including noise, using Planck functions at the specified source temperatures as model inputs. The modeled interferograms were then processed with identical processing steps used to compute the measured NESR. The NESRs shown in Fig. 6 were smoothed by a moving average function approximately $20 \mathrm{~cm}^{-1}$ wide.

Fig. 6 shows excellent agreement between the modeled and actual NESR results. The GIFTS EDU NESR requirements and objectives are also shown in Fig. 6. The measured NESR is well below the EDU requirement for both bands and near the EDU objective for the entire SMWIR band and most of the LWIR band. The EDU NESR objective is the original flight specification. As shown in Fig. 6, the GIFTS EDU has already demonstrated NESR levels that are at or near the levels required for a flight unit to provide the radiometric uncertainty required for atmospheric sounding. We are confident that from the experience gained with the GIFTS EDU, improvements can be made that will result in even better NESR performance for a flight instrument. 

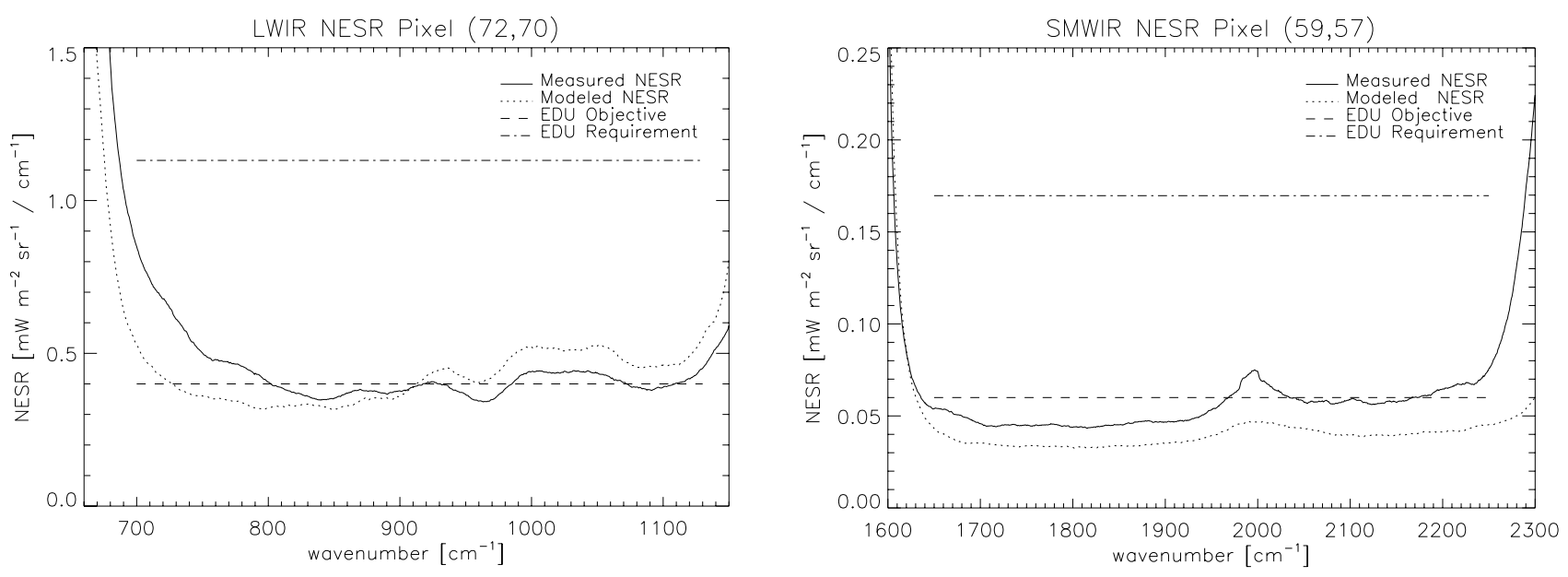

Fig. 6. Comparison of GIFTS EDU measured and modeled NESR for nominal pixels from the LWIR and SMWIR FPAs.

Fig. 7 shows the modeled and preliminary measured instrument line shape (ILS). A $\mathrm{CO}_{2}$ laser was used to produce the data for the measured ILS. To calculate the measured ILS several interferograms from an LWIR pixel were zero padded by a factor of 32 prior to Fourier transformation and the spectral average of the individual phase corrected spectra was computed. The spectrum was then peak normalized. No wavenumber scale correction was applied. To compute the modeled ILS, interferograms were generated using the sensor response model with an input spectrum consisting of a single spectral line calculated such that the modeled and measured lines are in the same position. The simulated interferograms were processed with the same processing steps used to compute the measured ILS. In this case the measured and modeled results are nearly identical.
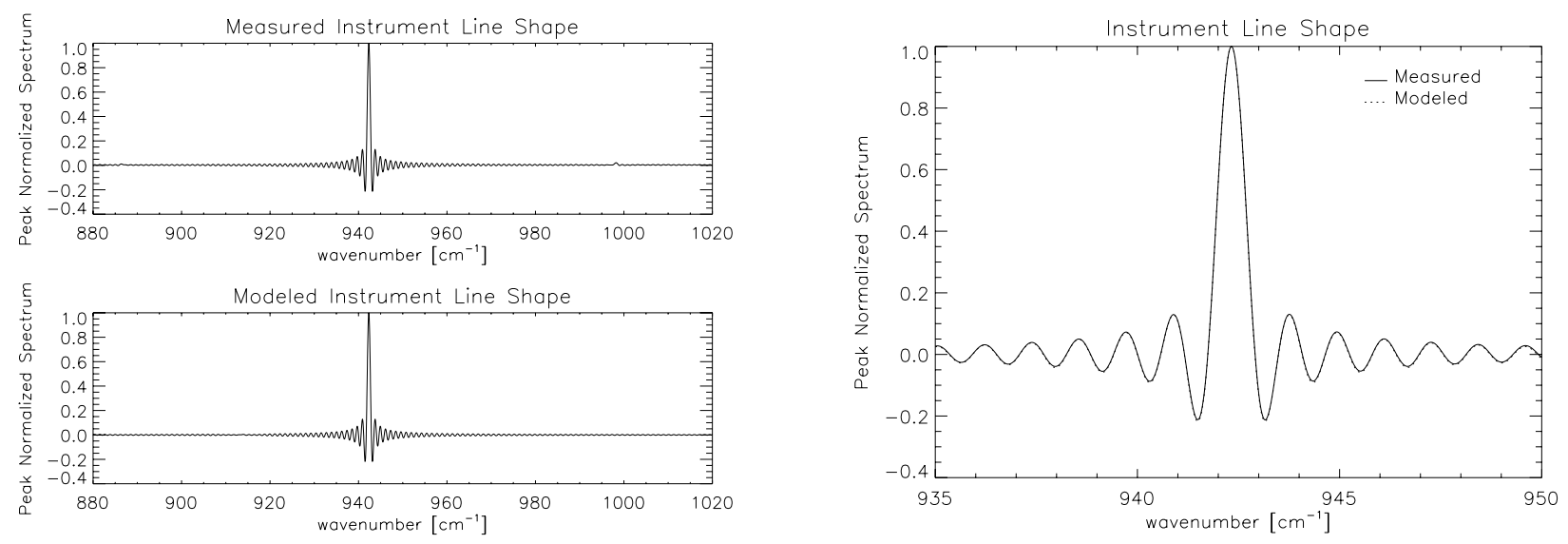

Fig. 7. Comparison of GIFTS EDU measured and modeled instrument line shapes.

Fig. 8 is a comparison between measured and modeled out-of-band harmonics that result from nonlinearities in the system, at two points in the dynamic range. The data used to produce the results in Fig. 8 were not processed by the DSP because the out-of-band harmonics would be filtered out. The measured results in Fig. 8 were calculated by computing average magnitude spectra for an LWIR pixel. The interferograms were truncated to produce spectra with approximately $8 \mathrm{~cm}^{-1}$ unapodized spectral resolution. The modeled results were calculated by simulating GIFTS interferograms, including nonlinearity, using Planck functions at the measured temperatures as the model input. Transmission through the MIC2 calibrator was also accounted for to simulate the actual conditions under which the data were collected. The simulated interferograms were processed identically to the measured interferograms to produce the modeled results in Fig. 8. There is reasonable agreement in the location and magnitudes of the predicted and actual 
nonlinear harmonics suggesting that the simulated nonlinearity is close to the actual GIFTS EDU system nonlinearity. This fact gives added confidence to the proposed and simulated method of linearity correction for actual GIFTS EDU interferograms.
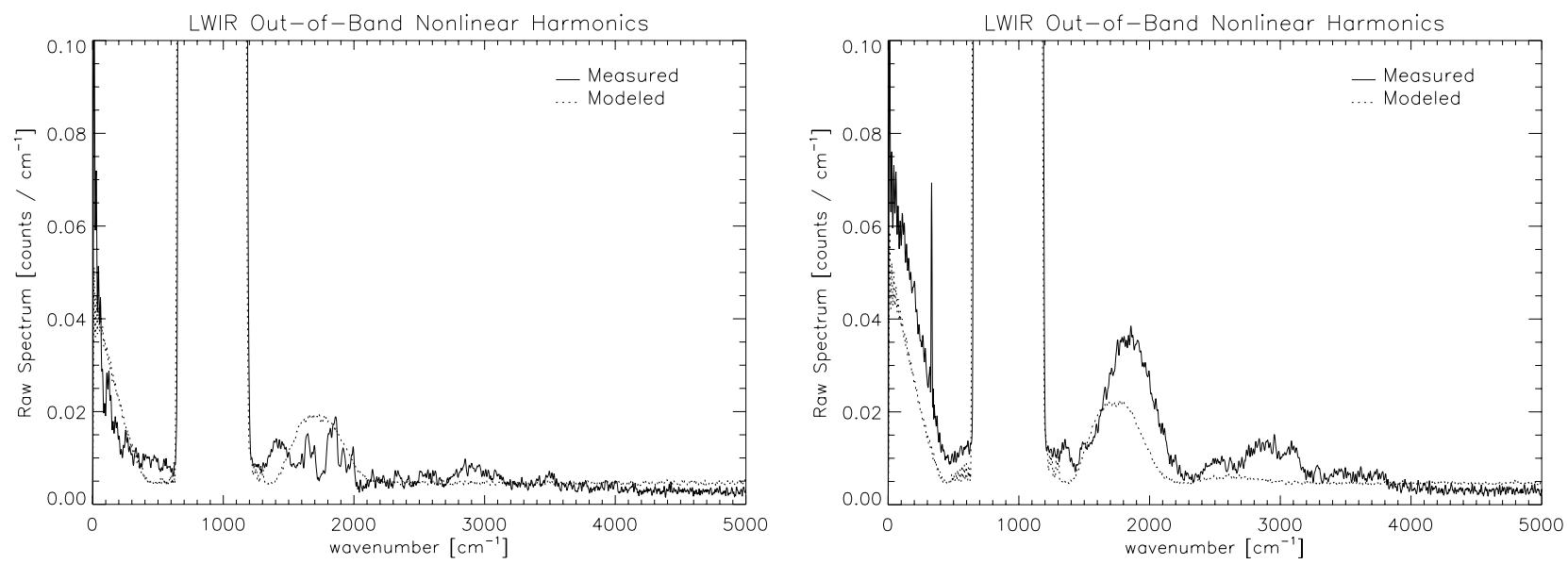

Fig. 8. Comparison of GIFTS EDU measured and modeled out-of-band nonlinear harmonics at two different points in the dynamic range. The measured data in the left plot are from one of the GIFTS EDU internal blackbodies at $286 \mathrm{~K}$. The measured data in the right plot were collected viewing an external blackbody at $350 \mathrm{~K}$ through the MIC2 calibrator in collimator mode.

Fig. 9 shows actual and modeled apparent spectral line shifts across the SMWIR FPA. In an imaging FTS there is an apparent shift in the position of a spectral line across the FPA that is caused by beams traveling through the interferometer at different angles. Once the apparent shifts have been measured, the spectral line positions can be corrected for. In Fig. 9 the actual spectral line shifts were calculated from measurements taken with a gas cell filled with $\mathrm{CO}$. Due to the experimental setup only a 15-pixel diameter portion of the FPA could be illuminated at once. The actual lines shifts across the FPA were calculated by analysis from measurements taken in the four corners and center of the FPA. The modeled line shifts were calculated by simulating GIFTS interferograms for an input spectrum consisting of a single spectral line and computing the ratio of the apparent to the true position of the spectral line for each pixel in the FPA. The actual results are in excellent agreement with the predicted results. The actual center of the interferometer is approximately 6.2 pixels above the center of the SMWIR FPA.

Apparent Line Shift (Actual)
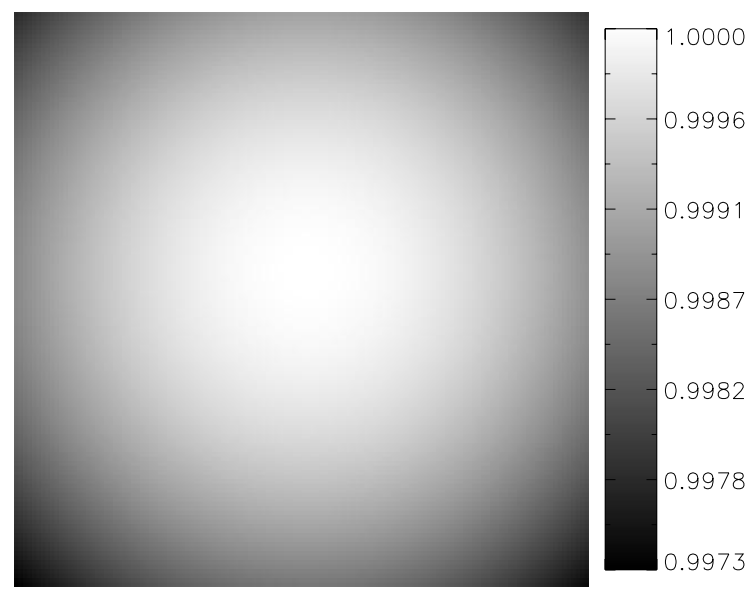

Apparent Line Shift (Modeled)

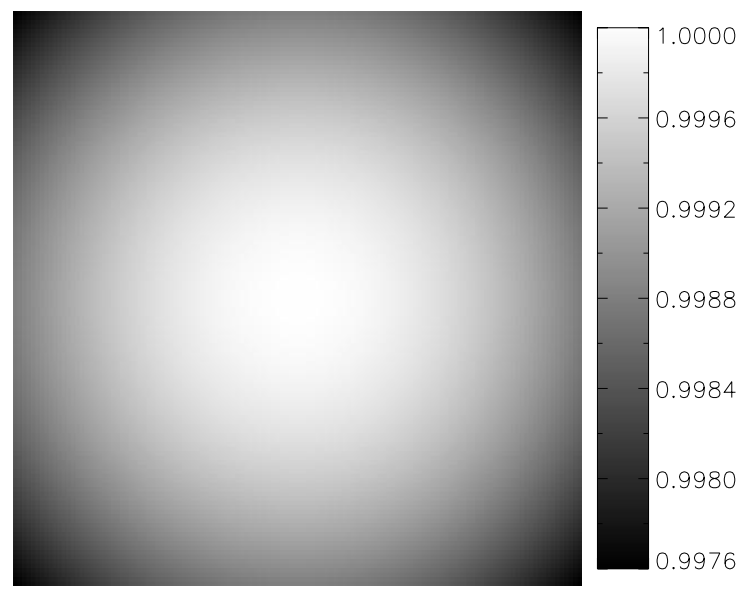

Fig. 9. Comparison of GIFTS EDU actual and modeled line shifts across the FPA. 


\section{SUMMARY}

We have presented a GIFTS sensor response model that has been shown to be in excellent agreement with preliminary GIFTS EDU measured results. The GIFTS EDU exhibits exceptional performance as evidenced by the preliminary data presented here. The validation of the sensor response model demonstrates that the GIFTS EDU performs substantially according to design. The NESR for the GIFTS EDU, a critical performance metric, easily meets the GIFTS EDU requirements and is at or near levels required for a flight instrument. More detailed analysis of the data collected during thermal vacuum testing and calibration is underway.

\section{ACKNOWLEDGEMENTS}

The GIFTS EDU was funded under federal contract NAS1-00071.

\section{REFERENCES}

1. Smith, W.L., D.K. Zhou, F.W. Harrison, H.E. Revercomb, A.M. Larar, H.L. Huang, and B. Huang, "Hyperspectral remote sensing of atmospheric profiles from satellites and aircraft," Proceedings of SPIE, vol. 4151, pp. 94-102, 2001.

2. Elwell, J.D., G.W. Cantwell, R.W. Esplin, G.B. Hansen, S.M. Jensen, M.D. Jensen, S.B. Brown, L.J. Zollinger, V.A. Thurgood, M.T. Esplin, R.J. Huppi, G.E. Bingham, H.E. Revercomb, F.A. Best, D. Tobin, J. Taylor, W.L. Smith, R. Riese, and R. Hooker, "A Geosynchronous Imaging Fourier Transform Spectrometer (GIFTS) for hyperspectral atmospheric remote sensing: instrument overview \& preliminary performance results," Proceedings of SPIE, vol. 6297.

3. Best F.A., H.E. Revercomb, R.O. Knuteson, D.C. Tobin, S.D. Ellington, M.W. Werner, D.P. Adler, R.K. Garcia, J.K. Taylor, N.N. Ciganovich, W.L. Smith, Sr., G.E. Bingham, J.D. Elwell, and D.K. Scott, "The Geosynchronous Imaging Fourier Transform Spectrometer (GIFTS) on-board blackbody calibration system," Proceedings of SPIE, vol. 5655, Multispectral and Hyperspectral Remote Sensing Instruments and Applications II, pp. 77-87, 2005.

4. Knuteson R.O., F.A. Best, G.E. Bingham, J.D. Elwell, H.E. Revercomb, D.C. Tobin, D.K. Scott, J.K. Taylor, and W.L. Smith, Sr., "On-orbit calibration of the Geosynchronous Imaging Fourier Transform Spectrometer (GIFTS)," Proceedings of SPIE, vol. 5655, Multispectral and Hyperspectral Remote Sensing Instruments and Applications II, pp. 66-76, 2005.

5. Wyatt, C.L., Radiometric Calibration: Theory and Methods, chp. 1, Academic Press, New York, 1978.

6. GIFTS SM EDU Instrument Calibration Plan, Technical Report SDL/01-005, Space Dynamics Laboratory, 2006.

7. SPIRIT III Ground Calibration Equipment Characterization Report, Technical Report SDL/94-073, Space Dynamics Laboratory, 1995.

8. HAES-15 Design and Performance Report, Technical Report SDL/03-217, Draft, Space Dynamics Laboratory. 